\title{
Lightweight Design Needs Support
}

The desire for the "right materials in the right place", so often repeated in automotive construction, is as justified as it is difficult to fulfil. The material parameters vary too much as they may be suitable in one use case and totally unsuitable in the next. It is not only material properties that play a role in enabling sound decisions to be made in favour of various metallic or plastics-based solutions. Decisive factors also include questions of availability, performance in production and recyclability. The latter aspect is gaining importance in times of ever scarcer resources.

Steel still holds on to its dominant position in the volume segment. Nevertheless, lightweight aluminium is continuously winning new friends in design and development. In this edition of lightweight design we focus on developments in steel, aluminium and also magnesium as materials for lightweight design that can compete with reinforced plastics.

Authors from the Fraunhofer Institute for Machine Tools and Forming Technology in Stuttgart investigate use of high-strength steels in reduced-thickness walls and their disadvantages for production engineering as well as possible solutions for the use of magnesium and highstrength aluminium alloys.

An article from Constellium describes, amongst others, how 7000 series aluminium alloys, which are ultra-high-strength but difficult to form, are gaining a foothold in the automotive sector and how they offer, in comparative terms, "the best compromise between weight-saving potential and cost".

Despite all the outstanding successes that lightweight car design has made possible - it cannot achieve everything on its own. A modern mix of materials alone will not cause the emission figures prescribed for 2020 of $95 \mathrm{~g}$ of $\mathrm{CO}_{2}$ per kilometre being met. Not even with the powertrain concepts deployed so far, even if they are further optimised. On the contrary, passenger vehicles are growing ever larger and tending to weigh more despite lightweight materials. The automotive industry is preventively complaining about stricter threshold values, however continues to focus on performance and driving enjoyment.

Autonomous driving will remain something of a pipe dream for a while partly due to unanswered questions concerning IT safety and legal issues, and electromobility is making little headway due to insufficient battery capacities and poor charging infrastructure. There is significant demand for new types of concepts for transportation in which lightweight design is not expected to bear all the expectations with regard $\mathrm{CO}_{2}$ reductions. On a side note, it would be possible for example to "recycle" a demand from our "environment party" from former times, namely whether a more relaxing speed limit could reduce emissions (and adrenaline levels) - even if the Greens no longer talk about it. 4 